\title{
Actividades que contribuyen al conocimiento de la Historia Local en estudiantes de noveno grado del ESBU Mártires de la Sierpe
}

\author{
Activities that contribute to the knowledge of Local History in ninth grade students of ESBU \\ Mártires de la Sierpe
}

\author{
Isolidia Hernández Torrez ${ }^{1}$ \\ Maité Felicia Valdivia Valdés ${ }^{2}$ \\ Eddy Yoel Díaz Martínez. Profesor ${ }^{3}$
}

\section{Resumen}

Este artículo ha abordado actividades que contribuyen al vínculo de los conocimientos de Historia Local con los de Historia de Cuba, a tenor de las necesidades objetivas descubiertas en los estudiantes de gno. grado de la ESBU Mártires de la Sierpe, consecuencia de la aplicación de diversos métodos teóricos, empíricos y matemáticos, reveladores también de las potencialidades que evidencian los educandos de estas edades, en cuyo modelo actual se apoya la investigación. La aplicación de los instrumentos de referencia puso en claro la necesidad de darle tratamiento a la Historia Local insertándola dentro de la Historia Nacional a través del programa de Historia de Cuba de gno grado a partir de la realización de actividades dinámicas y desarrolladoras, con lo cual se verifica las transformaciones que se decidieron lograr en los indicadores planteados.

Palabras clave: Actividades; historia local; historia de Cuba.

\section{Abstract}

This article has addressed activities that contribute to the knowledge link of Local History with Cuban History, in accordance with the discovered needs in the students of 9th grade at ESBU Mártires de la Sierpe, as a consequence of the application of various theoretical, empirical and mathematical methods, also revealing of the potentialities that the students of these ages show, in whose current model the research is based. The application of the reference instruments made it clear the need to treat Local History by inserting it into National History through the gth grade Cuban History program through dynamic and developmental activities, thereby the transformations that were decided to achieve in the proposed indicators are verified.

$1 \mathrm{MsC}$. Isolidia Hernández Torres. Coordinadora de Posgrado. Instructor. Universidad “José Martí Pérez”. Correo: isolidia@uniss. edu.cu; https://orcid.org/oooo-ooo1-8644-2843

2 MsC. Maité Felicia Valdivia Valdés. Subdirectora Docente Profesora Auxiliar. Correo: maite@uniss.edu.cu; https://orcid. org/oooo-0oo2-7924-9655

3 Lic. Eddy Yoel Díaz Martínez. Coordinador de Extensión Universitaria. Instructor. Correo: eddydm@uniss.edu.cu; https://orcid. org/oooo-0001-9879-5478

Recibido: 08/01/2021 - Aprobado: 05/02/2021 
Keywords: Activities; Local History; Cuban History.

\section{Introducción}

El sistema educacional cubano, eje central de la formación ideo-política, posee como fuerza motriz a la escuela encargada de formar en los estudiantes valores patrióticos que se sustentan en la cubanía, en el amor imperecedero a los héroes y mártires, en el conocimiento de las raíces históricas, para lo cual se han facilitado valiosas fuentes como soporte al maestro en su labor pedagógica.

El programa de la asignatura Historia de Cuba de gno. grado, a través del conocimiento propio de la Historia Patria, es una significativa vía mediante la cual se le da salida a una de las direcciones educativas, el trabajo político ideológico, núcleo central de todas las actividades de la enseñanza en Cuba. Posee 200 horas clases distribuidas en cuatro unidades temáticas, tiene entre sus objetivos desarrollar valores, modos de actuación y una cultura general derivadas de la significación que encierra cada proceso, suceso o personalidad destacada a lo largo de los siglos; propiciar en los estudiantes un desarrollo político ideológico de manera que experimenten sentimientos de compromiso y afecto por la revolución y sus principales figuras; sientan un profundo rechazo hacia el imperialismo y refuercen su amor a la soberanía y la independencia; contribuir además con su educación estética y con el uso adecuado de la lengua materna; así como vincular la Historia Nacional con la Historia Local, lo cual fortalecerá su apego e identificación con la Historia Patria al sentirse partícipes de ella.

El estudio de la Historia Local constituye un medio pedagógico para desarrollar motivos de estudio donde el alumno juegue un papel protagónico, busque y redescubra la historia del lugar donde vive. Sobre este particular se plantea en la Junta de Superintendentes de la Isla de Cuba (1926, p. 252), “...el conocimiento de la Historia Local, en sus hechos más sobresalientes debe preceder y servir de base al de la Historia Nacional".

Para dar tratamiento al tema de la Historia Local es preciso tener en cuenta los puntos de vista de destacados pedagogos cubanos, que desarrollaron su obra desde finales del siglo XVIII, cuyo pensamiento está vigente en los momentos actuales, entre los que se destacaron: Félix Varela (1785 - 1853), José de la Luz y Caballero (180o - 1862), Enrique José Varona (1849 - 1933) y José Martí (1853 -1895), los cuales nos dejaron una herencia de la que somos depositarios y continuadores.

Muchos investigadores han insistido en la importancia del vínculo de la Historia Nacional con la Historia Local. Algunas personalidades del territorio se han interesado por el conocimiento y enseñanza de la Historia Local, y han logrado publicar libros, artículos y otros documentos importantes que han facilitado que salgan a luz pública lo que se mantiene oculto bajo las piedras, se cuenta entre ellos con los maestros 
Carlos Jorge Meneses Meneses, Carlos Hernández Hernández, el director del museo municipal Reinaldo Pérez Jiménez y la licenciada Ileana Albelo, historiadora de dicha instalación.

A partir del análisis del banco de problemas de las Escuelas Secundarias Básicas Urbanas (ESBU), específicamente de la escuela "Mártires de La Sierpe", la observación empírica y la aplicación de diversos instrumentos de investigación científica se corroboraron deficiencias educativas en el área de historia nacional y local.

A partir de los resultados anteriores se declara como objetivo del trabajo: aplicar actividades de Historia Local vinculadas al programa de Historia de Cuba, encaminadas a perfeccionar el conocimiento en los estudiantes de gno. grado de la ESBU Mártires de la Sierpe.

\section{Revisión de literatura}

La enseñanza de la Historia como han expresado diferentes pedagogos y filósofos influye notablemente en la vida del hombre, de la sociedad, contribuye al desarrollo del intelecto de los estudiantes y propicia el amor patrio.

La Historia Local, en su generalidad, comprende personalidades, lugares y objetos que reflejan las vivencias de los antepasados y sus valores sobreviven en las nuevas generaciones gracias a la transmisión de los conocimientos que la escuela les aporta desde los estudios locales en los diferentes programas.

Todos los pueblos poseen sitios de interés local que constituyen un motivo bien justificado de orgullo. Hay valores cuya importancia es tal que trascienden sus fronteras. El conocimiento de la Historia Local es un deber insoslayable de la educación, así como un derecho inalienable de los pueblos conocer el acervo histórico de su localidad que ha acumulado del pasado, lo que se tiene en el presente, y lo que dejaremos a las futuras generaciones, para que aprendan de él, lo acrecienten y lo disfruten. En la concepción más divulgada, localidad es un vocablo del lenguaje cotidiano, concebido como calidad de las cosas que las determina a un lugar fijo: lugar o pueblo. Local, sitio o paraje cercado.

Existen otras definiciones vinculadas con las clasificaciones taxonómicas establecidas por distintos investigadores y que se refieren al estudio de los complejos territoriales naturales, que presentan distintas estructuras y comprenden diferentes áreas. De acuerdo con estos criterios, "localidad" es un complejo natural genéticamente homogéneo, que se ha formado como resultado de la asociación de comarcas dinámicamente interrelacionadas, que se difunden de un mismo basamento geológico" (Mateos, 1984, p. 33). 
Contradictoriamente a este criterio no se puede dejar de reconocer que el maestro con sus estudiantes puede definir, así mismo, qué es localidad para el proceso de enseñanza-aprendizaje que diseña y dirige. Se puede pensar, entonces, en qué localidad es "aquel territorio con dimensiones específicas, apropiado y transformado por una población como resultado de un proceso de edificación histórico-social, culturalmente auténtico" (Leal, 2002, p. 23).

En nuestra propuesta consideramos la localidad como el "entorno más cercano a la escuela, puede ser un batey, una comunidad, un poblado, una ciudad o un municipio. Se considera en su máxima dimensión al municipio, debido a que en este nivel existe una clasificación de símbolos que deben ser trabajados por cualquier escuela enclavada en su jurisdicción". (Reigosa, 2003, p. 64)

\section{La Historia Local y su vínculo con la Historia Nacional. Sus características}

La Historia Local, se define como el estudio hecho por los estudiantes, bajo la orientación del maestro, de los hechos, fenómenos y procesos singulares y locales del pasado lejano o próximo y del presente de determinado territorio, en su relación con el devenir histórico nacional.

La Historia Local puede ser un productivo aprendizaje en la medida en que los estudiantes sean orientados a "buscar" para "encontrar" y después discutir. De lo que se trata es que la clase de Historia privilegie actividades en las cuales los estudiantes tengan que analizar y dar opiniones.

El profesor Díaz Pendás (1997) ha planteado que existen aspectos del pensamiento martiano que trazan pautas a la labor docente por la vigencia que poseen. Uno de estos aspectos es la labor de indagación por parte de los estudiantes del conocimiento. La participación de los estudiantes está vinculada a la concepción de un sistema de actividades y tareas docentes que le conduzcan a esa búsqueda y análisis reflexivo del conocimiento y del valor que tiene para él y la sociedad.

El maestro puede utilizar métodos orales como el relato, la descripción, la explicación, el diálogo y la conversación heurística. Por otra parte, los documentos que norman la enseñanza de esta disciplina establecen, además, el trabajo con materiales gráficos como el texto, documentos, mapas, pancartas, láminas y la interacción con softwares. Sin embargo, ¿han alcanzado nuestros maestros una competencia comunicativa que les permita relatar, contar los hechos teniendo en cuenta la importancia que tiene la palabra de ellos? ¿A pesar de la experiencia que tienen, dominan el trabajo con estos medios de enseñanza, los utilizan sistemáticamente en sus clases?

Existen fuentes del conocimiento histórico que no constituyen objeto de trabajo de los historiadores en su labor de investigación. Indiscutiblemente que el trabajo 
con las piezas museables constituye una de las principales fortalezas en la educación de la Historia Local. Es necesario, de hecho, conocer las alternativas metodológicas existentes para la correcta utilización de esta fuente del conocimiento histórico.

Un museo constituye un sistema de medios para la enseñanza de la Historia. Los museos de historia poseen valiosos objetos originales que constituyen su esencia, combinados armónicamente con fotos, mapas, esquemas, datos estadísticos, textos y otros medios gráficos portadores del mensaje al visitante. Aquí radica precisamente la concepción en sistema de todos estos medios. Entre las formas de trabajo con el museo más recomendables que podemos seguir están:

$>$ La visita dirigida o guiada.

$>$ El trabajo independiente del estudiante apoyado en la guía de observación.

$>$ La clase que se desarrolla en el museo.

$>$ La vinculación de lo aprendido en el museo con la clase de la escuela (Díaz Pendás, 2008, p. 10).

Sin embargo, vale apuntar que para ser efectivo el uso de este medio es necesaria la autopreparación profunda del maestro de manera que la clase, visita u observación sean realizadas con calidad y adquieran verdadero significado para los estudiantes.

\section{Para vincular lo nacional con lo local existen cuatro formas:}

$>$ Lo local como lo nacional.

Lo local como reflejo de lo nacional.

Lo local como peculiaridad de lo nacional.

Lo local como inserción de lo nacional. Cada una de estas variantes con sus propias particularidades (Díaz Pendás, 2008, p. 25).

Por ello, los maestros deben dominar los contenidos locales y buscar las vías más adecuadas para que sus estudiantes piensen y reflexionen utilizando fundamentalmente la investigación. Deben definirse qué fuentes del conocimiento potencian habilidades del pensamiento lógico, así como, las actividades para los sistemas de clases.

\section{El enfoque histórico - cultural de Vigostki (1983) en el contexto de la Historia Local}

Vigostki (1983) dio gran importancia a la relación entre el aprendizaje y el desarrollo, para esto se ha basado en el concepto de zona de desarrollo próximo (ZDP) que es la distancia entre el nivel real de desarrollo del individuo expresado en forma espontánea y el nivel de desarrollo manifestado gracias al apoyo de otra persona. 
Este trabajo tiene su punto de partida en el enfoque histórico - cultural y la teoría de la actividad, en tanto explica con claridad cómo el proceso educativo se puede convertir en lo más importante, utilizando un sistema de relaciones interpersonales y de medios lo más interrelacionado al estudiante para incentivar su motivación e interés por la resolución de las tareas docentes, que implica la preparación política y científica para el desarrollo exitoso del proceso educativo, con el protagonismo que caracteriza al estudiantado contribuyendo a perfeccionar el proceso de asimilación del conocimiento.

\section{Materiales y métodos}

Este estudio, el cual corresponde a los resultados del trabajo científico metodológico, utilizando el método de investigación acción colaborativa, se realiza específicamente en el municipio La Sierpe, en la provincia de Sancti Spíritus. Esta escuela posee tradición en los estudios de la localidad y ha tenido resultados. Por ello se hace necesario trabajar, desde las Ciencias Pedagógicas, en la búsqueda de vías que permitan una incidencia coherente, en función de la Historia de Cuba y el vínculo con la Historia Local, de las diversas influencias educativas que recibe este alumno durante su formación básica y que sea extensiva para la cultura general integral y como experiencia significativa en la educación de las nuevas generaciones.

\section{Resultados y discusión}

La experiencia de los autores en el trabajo sistemático en los estudios locales, así como en la tutoría de Trabajos de Curso, Diplomas y la aplicación de diferentes instrumentos relacionados con las temáticas objeto de estudio de esta investigación, ha permitido determinar la existencia de problematizaciones que pueden ser solventadas por la vía de la investigación, dentro de ellas están:

Los estudiantes no reconocen las manifestaciones de lucha en el territorio en diferentes momentos de la historia; Es insuficiente el dominio que poseen acerca de las personalidades del territorio que se destacaron en los diferentes momentos de la lucha revolucionaria; no logran establecer la relación adecuada entre los hechos y la etapa de la Historia de Cuba en que sucedieron, así como con las personalidades de la Historia Nacional con las que se relacionan; No se identifican, ni manifiestan expresiones de apego a la Historia de su entorno local. No muestran interés por el conocimiento de hechos históricos locales, ni por la participación en actividades que contribuyen al cuidado y conservación de tarjas y monumentos históricos locales.

A partir de las deficiencias, las actividades se formulan sobre la base de estas y aprovechando las potencialidades que poseen los estudiantes, pues estos poseen conocimientos de los contenidos fundamentales de la Historia Nacional y sus etapas, son educandos entusiastas, dinámicos e interesados por la realidad que los rodea, 


\section{CIENCIAS SOCIALES}

flexibles a motivaciones que contribuyan a elevar su nivel cultural, además son individuos nacidos y formados sobre la base de los sólidos principios de la sociedad socialista siendo fieles exponentes de los ideales patrióticos en los que se sustenta la Revolución Cubana.

Por todo lo antes expuesto se evidencia la necesidad de aplicar una propuesta de solución que permita erradicar la problemática antes planteada.

\section{Fundamentación teórica de las actividades para el conocimiento de la Historia Local}

Al consultar a Leontiev (1987), este define la actividad del conocimiento como el "proceso de interacción sujeto-objeto, dirigido a la satisfacción de las necesidades del sujeto, como resultado del cual se produce una transformación del objeto y del propio sujeto" (p. 41).

La concepción de la actividad es un aspecto medular en la teoría histórico - cultural, desarrollada por Leontiev (1987) en la que se deja claro que es en la actividad, donde se forman y desarrollan los procesos psíquicos y las cualidades de la personalidad. La actividad está conformada por dos componentes: los intencionales y los procesales. Los primeros le dan intención, dirección, orientación y finalidad a los segundos, que constituyen la manifestación y expresión del propio proceso de la actividad.

Los componentes estructurales de la actividad son: su objeto, su objetivo, su motivo, sus operaciones, su proceso y el sujeto que la realiza. Los componentes funcionales de la actividad son la parte orientadora, la parte de ejecución y la parte de control, las que se encuentran interrelacionadas íntimamente. Atendiendo a lo anterior las actividades que se proponen presentan las siguientes características:

Elevar en los estudiantes el conocimiento de la Historia de la localidad.

Lograr con el desarrollo de cada actividad formar modos de actuación.

Las actividades están en plena correspondencia con los documentos que norman el trabajo metodológico de la Historia de Cuba.

La propuesta se considera novedosa porque tras realizar una adecuada selección de los principales hechos y personalidades del entorno local, logra su inserción al proceso de enseñanza aprendizaje de la Historia de Cuba.

Están concebidas para ser desarrolladas en el proceso docente-educativo, utilizando variadas formas de organización del mismo. Para el desarrollo de las actividades se plantea su realización de forma práctica, lo que permitirá a los estudiantes apropiarse del sistema de conocimientos planteados. 
Las actividades parten de la relación existente entre los contenidos de la Historia Nacional incluidos en el Programa de gno.grado con los hechos acontecidos en la localidad. Estas están en correspondencia con la edad y las características de los estudiantes y materializa el objetivo del trabajo porque contribuyen al conocimiento de la Historia Local.

En la implementación de las actividades se cumplen las funciones de dirección que articulan con diferentes fases, como las siguientes:

- La planificación: permite prever y organizar dentro del sistema de evaluación un adecuado proceder sistemático y coherente.

- Organización: posibilita el cumplimiento de cada una de las actividades por el investigador que ejecutará la evaluación, lo que contribuirá en los estudiantes al conocimiento de la Historia Local logrando sentimientos de identificación y apego hacia ella.

- Control: mide la efectividad del proceso de educación y el cumplimiento de los objetivos trazados para potenciar el conocimiento de la Historia Local.

\section{Propuesta de actividades a modo de ejemplo se ilustra con algunas seleccionadas.}

\section{Actividad 1}

Título: "La Toma de Agua: Un legado de los agro- ceramistas"

Objetivo: Caracterizar al grupo aborigen asentado en Toma de Agua.

Acción: Tarea investigativa.

\section{Operaciones:}

El grupo aborigen de Toma de Agua, constituye el sitio neolítico más integralmente investigado de la región arqueológica de la provincia de Sancti-Spíritus. Sobre él investigue.

- Ubicación geográfica y condiciones naturales del sitio.

- Actividades económicas de subsistencia a partir de los restos fáunicos encontrados.

- Tradición alfarera que poseían.

- Artefactos estructurales que poseían que han sido hallados en este sitio.

- Tipo de herramientas utilizadas (cantidad de cada una de las halladas en estudios arqueológicos realizados).

- Conclusiones acerca de su nivel de desarrollo. 


\section{Conclusiones}

Para el desarrollo de esta actividad se orientó a los estudiantes en clases anteriores realizar la actividad como tarea en la biblioteca municipal mediante el estudio del texto citado. La mayor parte de los estudiantes se apropió de las características fundamentales que serían debatidas en la clase, lo que posibilitó el desarrollo con calidad de las operaciones concebidas, logrando caracterizar el grupo aborigen asentado en Toma de Agua.

\section{Bibliografía:}

Pérez Jiménez, R. et al. Arqueología aborigen del sitio de Toma de Agua. p 9-3o.

\section{Actividad 2}

Título: En el lugar que merece.

Objetivo: Valorar la personalidad de Néstor Leonelo Carbonell Figueroa.

Acción: Tarea extraclase.

\section{Operaciones:}

“No por ser Néstor L. Carbonell, carne y orgullo nuestro, hemos de ponerlo sobre catedrales y montañas. Tampoco hemos de colocarlo, por este único motivo, por debajo de los álamos y a la altura de la hierba inútil” (Carbonell, 2007, p. 5)

- Con el propósito de elevarlo a la altura que sus valores morales y políticos exigen, sin caer en localismos, te invitamos al análisis de algunas reflexiones que destacadas personalidades han realizado sobre Néstor L Carbonell.

\section{José Martí:}

Vive en Tampa como un padre de pueblo, el fidelísimo cubano Néstor L Carbonell. Él es de aquellos cubanos incansables que solo sienten dicha en lo que eleve y mejore el alma patria, en que entre los cubanos y los hombres todos cunda el patriotismo y el cariño, en llevar a los rincones más dormidos la buena voz cubana (Ferrer Carbonel, 2005, p. 2).

Néstor Carbonell y Rivero: “(...) ejemplo vivo de austeridad acrisolada, predicador de que es mejor quemar la casa que abrirla a la deshonra (...)” (Ferrer Carbonel, 2005, p. 3) Wen Gálvez: 
No puede decirse que pertenezca a la generación pasada, porque a la verdad, a esta generación también le pertenece. Si así no fuera, ¿por qué entonces la juventud lo busca y lo oye? Tiene figura de apóstol y su vida es un apostolado. Su semblante risueño, tranquilo y resignado, así lo indica, y sus hechos así lo atestiguan. A veces escudriño en su mirada, y quiero ver decaimiento o desmayo, y siempre fracaso (...) (Ferrer Carbone, 2005, p. 91).

\section{Carrosquilla Mallarino:}

Su historia de patriota, obsesionado de libertad, lo fraterniza en páginas ilustres con la evangélica memoria del tres veces glorioso José Martí y lo exalta a mis ojos enamorado de los grandes sueños como a un paladín, en cuya palabra ferviente reviven los días de angustia y de heroísmo de la ínsula hermosa, con mayor emoción que en las páginas de algún libro" (Ferrer Carbonel, 2005, p. 126).

Tomando como referencia estas reflexiones y la invitación de consultar otras fuentes que ahondan en la vida y obra de este patriota. Valora su personalidad. Conclusiones: Esta actividad les dará la posibilidad a los estudiantes de valorar la personalidad de Néstor Leonelo Carbonell ubicándolo así en el lugar que merece dentro de la Historia, para ello le facilitamos las reflexiones que

Importantes personalidades han hecho sobre él, así como la invitación a consultar otras fuentes que harán su tarea más profunda y fructífera.

\section{Bibliografía}

Ferrer Carbonell, O. (2005). Néstor Leonelo Carbonell como el grito del águila. La Habana, Cuba: Editorial de Ciencias Sociales.

Carbonell Rivero, N. L. (2007). Resonancias del pasado. Sancti-Spíritus, Cuba: Ediciones Luminaria.

\section{Desarrollo y evaluación de la aplicación de las actividades}

Las actividades propuestas para transformar la realidad del objeto de estudio, permitiendo comprobar los resultados iniciales y finales con la muestra seleccionada, en este sentido se determinaron las fases siguientes:

- Fase de diagnóstico: permitió profundizar sobre el tema a partir de la aplicación y revisión de diferentes instrumentos a los estudiantes de gno. grado, con el objetivo de comprobar las carencias y las potencialidades que presentan respecto al conocimiento de la historia local. 


\section{CIENCIAS SOCIALES}

- Fase formativa: se aplicó la propuesta de las actividades de los estudiantes de 9no. grado con el objetivo de la adquisición de conocimientos sobre la Historia Local.

- Fase de control: se utilizó para constatar la efectividad del trabajo aplicándose nuevamente una serie de instrumentos a los estudiantes para comprobar la efectividad de dichas actividades y si estas lograron contribuir al conocimiento de la Historia Local y modificar por tanto el nivel de dichos conocimientos.

\section{Principales indicadores trabajados}

- Conocimiento sobre los aspectos importantes sobre la Historia de la localidad.

- Reconocimiento de las manifestaciones de lucha en el territorio en diferentes momentos de la historia.

- Dominio de las personalidades del territorio que se destacaron en los diferentes momentos de la lucha revolucionaria.

- Establecer la relación adecuada entre los hechos y la etapa de la Historia de Cuba en que sucedieron, así como con las personalidades de la Historia Nacional con las que se relacionan.

- Identificación y apego con la historia de su entorno local.

- Interés por el conocimiento de hechos históricos locales.

- Participación en el cuidado y conservación de tarjas y monumentos históricos locales.

Los instrumentos aplicados en un momento inicial corroboraron el desinterés de los estudiantes por el conocimiento de hechos y personalidades históricos locales, así como su escasa motivación ante visitas a tarjas y monumentos que contribuyeran a fomentar su conocimiento acerca de la historia acontecida en el lugar donde viven. En un segundo momento, es decir, tras la aplicación de las actividades propuestas en el trabajo, se evidenció en el $100 \%$ un crecimiento en el interés de los estudiantes por conocer el aporte de su localidad a la Historia Nacional y su marcado interés en revivir la misma a través de la visita a tarjas, monumentos y al museo local.

\section{Conclusiones}

La revisión bibliográfica permitió determinar los principales fundamentos teóricos y metodológicos que sustentan los conocimientos de la Historia Local a través del proceso educativo de Historia de Cuba en estudiantes de gno. grado.

El diagnóstico del estado real de los conocimientos de la Historia Local en estudiantes de 9no. grado de la ESBU "Mártires de La Sierpe" permitió concluir que este se comporta con niveles bajos en cuanto a los indicadores de las dimensiones cognitiva y afectiva. 
Las actividades asociadas al proceso de enseñanza aprendizaje de Historia de Cuba que contribuyen al conocimiento de la Historia Local en estudiantes de gno. grado de la ESBU "Mártires de La Sierpe", se caracterizaron por ser creativas, dinámicas, desarrolladoras en la educación de la Secundaria Básica donde los estudiantes tienen un papel protagónico.

Después de aplicar las actividades se elevó a un nivel alto el conocimiento de la Historia Local en estudiantes de 9no.grado de la ESBU "Mártires de La Sierpe" en cuanto a los indicadores de las dimensiones cognitiva y afectiva.

\section{Lista de referencias}

Carbonell Rivero, N. L. (2007). Resonancias del pasado. Sancti-Spíritus, Cuba: Ediciones Luminaria.

Díaz Pendás, H. (1997). En pos de un encuentro cercano con Clío. Revista Desafío escolar. 3(1), 41-47.

Díaz Pendás H. (2008). El museo vía para el aprendizaje de la Historia. La Habana, Cuba: Editorial Pueblo y Educación.

Ferrer Carbonell, O. (2005). Néstor Leonelo Carbonell como el grito del águila. La Habana: Editorial de Ciencias Sociales.

Junta de Superintendentes de la Isla de Cuba (1926). Cursos de estudios para las escuelas públicas. La Habana, Cuba: (s. n.).

Leal, H. (2002). Pensar, reflexionar y sentir en las clases de Historia. La Habana, Cuba: Editorial Pueblo y Educación.

Leontiev, A. N. (1987). Actividad, conciencia y personalidad. La Habana, Cuba:Editorial Pueblo y Educación.

Meneses, Meneses, JC. (2008). Historia de La Sierpe. En Proceso de Edición

Mateos Torneos, J. (1984). Tipos históricos de la unidad del conocimiento científico. La Habana, Cuba: Editorial Ciencias sociales. Meneses, Meneses, C. J. (s. f.). Apuntes. (Sin editar).

Pérez Jiménez, R. et. al. (2007). Arqueología aborigen del sitio Toma de Agua. Ediciones Luminarias: Sancti Spíritus, Cuba. 


\section{CIENCIAS SOCIALES}

Reigosa Lorenzo, R. (2003). Estrategia de superación profesional de los docentes de secundaria básica para la formación del valor patriotismo en sus estudiantes. (Tesis de doctorado inédita). Universidad de Ciencias Pedagógicas Capitán Silverio Blanco Núñez, Sancti Spíritus, Cuba.

Vigostky, Lev (1983). Zona de Desarrollo Próximo. Disponible en: https://www.actualidadenpsicologia.com/que-es/zona-desarrollo-proximo/ 